\title{
El estudiante chino de español como lengua extranjera (ELE) Diálogo entre la cultura china y el contexto educativo
}

\author{
Chinese students of Spanish as a Foreign Language. Dialogue \\ between the Chinese culture and the educational context
}

María del Carmen Azpiroz*

\begin{abstract}
Resumen
Es una visión extendida en la literatura sobre los estudiantes chinos en educación superior que sus estilos de aprendizaje están predeterminados por su cultura. La cultura china está influenciada por el confucianismo y la importancia asignada al aprendizaje memorístico y al libro como fuente casi exclusiva de conocimiento. Existen menos voces que exploren la posibilidad de que se genere un cambio significativo en los estilos de aprendizaje de los estudiantes chinos como consecuencia de realizar estudios en un nuevo contexto educativo con la exposición a metodologías alternativas de enseñanza y a diferentes tipos de evaluación.
\end{abstract}

Se interrogó a 42 estudiantes de una provincia del noreste de China, quienes se encuentran estudiando español como lengua extranjera en una universidad privada de Uruguay. El objetivo consiste en obtener datos sobre los posibles cambios en los estilos de aprendizaje de los estudiantes a partir de su llegada a Uruguay así como la valoración que hacen de esos cambios.

En términos generales, los estudiantes chinos que fueron interrogados señalan como aspectos positivos de su experiencia en Uruguay las metodologías de enseñanza más centradas en el estudiante, el aprendizaje independiente y el pensamiento crítico.

El artículo cuestiona la visión del estudiante chino pasivo, obediente, pegado al libro de texto, al docente y las estrategias de memorización y propone que dicho comportamiento puede ser producto de la influencia de los contextos educativos a los que dichos estudiantes han estado expuestos y de los requerimientos de aprobación de los cursos, y no únicamente de la influencia de valores culturales fuertemente arraigados.

PALABRAS CLAVE: Cultura, estilos de aprendizaje, estudiantes chinos, español como lengua extranjera, educación superior.

\section{Abstract}

It is an accepted view in the literature on undergraduate Chinese students that their learning styles and learning strategies are culturally predetermined. Chinese culture is influenced by Confucianism and the importance it places on roten memory and the book as an almost exclusive source of knowledge. There are only a few voices who explore the possibility that a change of Chinese learning styles and learning strategies could take place as a consequence of a new educational environment and the exposure of learners to alternative teaching methodologies and types of assessment.

Forty two students from a province located on Northeast China were interviewed while they 
were studying Spanish as a Foreign Language in a Uruguayan private University. The aim was to get data on possible changes in their learning styles since their arrival in Uruguay and on the evaluation they make on those changes.

Generally speaking, students who were interviewed identify as part of the positive aspects from their experience in Uruguay the student-centered teaching methodologies, independent learning and critical thinking

The article questions the view of Chinese students as passive, obedient, rote memorizers and stuck to the textbook and the teacher, and suggests that such behavior could be due more to the education context to which they have been exposed and the course requirements than to the exclusive influence of deeply rooted cultural values.

KEY WORDS: Culture, learning styles, Chinese students, Spanish as a Foreign Language, higher education.

\section{Introducción}

En los últimos años, el número de estudiantes internacionales ha aumentado rápidamente y Uruguay ha venido experimentando una creciente presencia de estudiantes de otros países y culturas. En el caso del trabajo con estudiantes asiáticos en general y chinos en particular, el escaso conocimiento en Uruguay sobre las características de dichos estudiantes puede fácilmente desviarnos hacia una visión estereotipada de los mismos (estereotipos que también se encuentran en la amplia literatura disponible sobre "el estudiante asiático").

Es una visión extendida en la literatura sobre los estudiantes chinos la afirmación de que sus estilos de aprendizaje están predeterminados por su cultura (Neuman y Beckerman, 2000; Chan, 1999; Spizzica, 1997). El constructo de estilos de aprendizaje ha recibido diversas denominaciones: estilos cognitivos, enfoques de aprendizaje, preferencias de aprendizaje, estilos intelectuales, por lo que se trata de una categoría integradora que caracteriza la manera habitual y preferida con que cada alumno percibe, interactúa y responde a los ambientes de aprendizaje (Dörney, 2005).

Chan (1999) cree que los estilos de aprendizaje de los estudiantes chinos están muy influenciados por el confucianismo y dominados por el aprendizaje memorístico generalmente descrito como aprendizaje superficial, sin comprensión y dependiente de la autoridad. Estas afirmaciones ocurren al mismo tiempo que los estudiantes chinos obtienen medallas de oro en ciencia y han llamado la atención por su excelente rendimiento académico (Tai Hau y Hou, 2010). En el informe mundial de 2009 del Programa para la Evaluación Internacional de Alumnos (PISA, según sus siglas en inglés), Shanghái obtuvo los resultados más altos en ciencias, matemáticas y lectura. No deberíamos sorprendernos si descubrimos que los enfoques de aprendizaje de los estudiantes chinos son más complejos y profundos de lo que parecen a primera vista.

Los estudiantes de culturas de herencia confucionista comparten un conjunto de valores y creencias sobre el aprendizaje que han sido transmitidos por generaciones. Li (2012) sostiene que conceptos como mente, razonamiento, investigación y conocimiento objetivo son raros en la jerga china del aprendizaje (a pesar de la reciente importación de muchos de estos conceptos desde Occidente). De manera similar, conceptos como virtudes del aprendizaje, esfuerzo personal, resistencia a las dificultades, perseverancia y humildad no son tan frecuentes en los textos paradigmáticos de la tradición occidental del aprendizaje.

Desde la época de Confucio (551 a. C. -479 a. C.), la civilización china ha dado mucho valor a la educación. El confucianismo fue adoptado como religión oficial durante la dinastía Han (250 a. C.) y los clásicos confucianos se transformaron en la base de los exámenes que, durante 
la China imperial (606-1905), sirvieron para seleccionar a los candidatos a funcionarios del gobierno. Los alumnos debían aprender los denominados "Cuatro Libros" y "Cinco Clásicos" de memoria. Si bien el examen fue abolido por la emperatriz Cixí en 1905, se considera que dicha tradición continúa hasta el día de hoy, si tenemos en cuenta por ejemplo el famoso y temible examen de admisión a la universidad conocido como gao kao. Se trata de un examen de tres días de duración en el que se evalúan los conocimientos adquiridos durante todo el transcurso educativo formal. La dificultad de este examen así como la presión familiar para obtener buenas calificaciones y entrar a una prestigiosa universidad han dado lugar a alarmantes casos de depresión entre los adolescentes. La predilección de los estudiantes chinos por el recitado y la repetición podría además estar relacionada, de acuerdo a Kember (2010), con el legado de la manera de aprender los caracteres chinos, los cuales son dominados justamente mediante numerosas repeticiones de los mismos.

Una visión opuesta a esta especie de predeterminación cultural está dada por autores como Kee-Kuok Wong (2004), quien sostiene que dicho concepto ha limitado nuestra posibilidad de comprender la complejidad de la experiencia internacional con estudiantes chinos. El propio investigador relata que, habiendo nacido en una familia china tradicional, podía ser considerado un "estudiante pasivo", absorbiendo todo lo que el profesor decía. Sin embargo, luego de mudarse a Australia, tras ser expuesto a diferentes métodos de enseñanza y haber sorteado algunos obstáculos, logró disfrutar de una enseñanza de corte constructivista. Littlewood (2000) sostiene que a pesar de ciertas dificultades iniciales, los estudiantes chinos se adaptan rápidamente a otros sistemas de aprendizaje y que esas dificultades son producto de experiencias previas (métodos tradicionales de aprendizaje, tipos de evaluación, número de estudiantes por aula) más que de factores culturales arraigados.

Cheng (2000) postula que los estudiantes chinos adoptan ciertos estilos de aprendizaje por motivos puramente pragmáticos: los exámenes tienen una estructura determinada y los estudiantes deben desarrollar los estilos que aseguren su éxito en los mismos. Kirkbride y Tang (1991) sostienen que cuando un estudiante percibe que la evaluación requiere adquisición pasiva y reproducción simple de conocimiento adoptará un enfoque superficial y empleará bajos niveles de estrategias cognitivas como la memorización. Cuando se percibe que la evaluación requiere un alto proceso cognitivo, como aplicar información a la solución de problemas, entonces los estudiantes serán más proclives a poner en práctica el enfoque profundo de aprendizaje.

A pesar de que muchas de estas afirmaciones pueden tener incluso un carácter intuitivo, existe muy poca literatura que explore la posibilidad de que se produzca un cambio en los estilos de aprendizaje de los estudiantes chinos de acuerdo al contexto. La visión general es que el bagaje cultural es un "chaleco de fuerza", una construcción muy difícil de modificar aun si el contexto cambia. Li (2012) argumenta que el contexto educativo puede contribuir muy poco al cambio de las creencias culturalmente informadas que tienen los estudiantes mucho antes de iniciar la educación formal. Los primeros años son esenciales para el futuro del niño, y durante esos años formativos los niños habrán desarrollado creencias y comportamientos con respecto al aprendizaje con los que vendrán el primer día de clases.

Con una visión diferente, Volet y Renshaw (1996) examinaron el cambio en los enfoques de aprendizaje de los estudiantes al comienzo y final del semestre. La muestra consistía en un grupo de estudiantes australianos y otro del sureste asiático, quienes se encontraban estudiando en una universidad de Australia. Los resultados indicaron que al final del semestre las diferencias en los enfoques de aprendizaje entre los dos grupos, que habían sido halladas al comienzo del semestre, habían desaparecido, fenómeno que atribuyeron a la adaptación 
de los estudiantes del sureste asiático a las demandas académicas del curso y al ambiente de aprendizaje. Conviene citar a Zhang et al. (2012) quienes reafirman que los estilos de aprendizaje son maleables como respuesta al contexto educativo.

"En vez de mantener visiones estereotipadas de los estilos intelectuales de diferentes grupos culturales, uno debería darse cuenta de que los estilos son maleables. Las personas que experimentan un choque cultural, deberían tener confianza en que es una cuestión de tiempo antes que su pensamiento se vuelva más efectivo en la nueva cultura de acogida" (2012, pág. 25).

Los estudiantes pueden tener preferencias por una u otra manera de aproximarse al aprendizaje pero estas predilecciones podrán ser llevadas a la práctica o no dependiendo del contexto de enseñanza, dentro de una interacción que no es muy diferente a la de la herencia y el medio.

\section{Aspectos culturales y contextuales del aprendizaje de los estudiantes chinos}

\subsection{Fuente y transmisión del conocimiento}

Scarcella (1990) sostiene que muchas culturas asiáticas ven al libro como fuente de todo el conocimiento y la sabiduría. El énfasis en el aprendizaje de los textos clásicos ha sido una característica de la civilización china a lo largo de su historia. Es frecuente encontrar en la bibliografía que los estudiantes chinos son pasivos receptores del conocimiento y que prefieren clases centradas en el docente, y esto hace que el aprendizaje comunicativo de lenguas extranjeras presente dificultades prácticas (Kirkbride y Tang, 1991).

Algunas investigaciones parecen corroborar esta hipótesis. Kember (2001) realizó 53 entrevistas a estudiantes de Hong Kong. La mayoría de estos estudiantes piensa que el rol del profesor es transmitir un cuerpo de conocimientos y su rol como estudiantes es absorber el conocimiento validado por el texto o por el profesor. El resultado del proceso de enseñanza y aprendizaje será juzgado de acuerdo a si los estudiantes son o no capaces de reproducir ese cuerpo de conocimientos para el examen.

Para autores como Nelson (1995) es justamente la obsesión por los exámenes, especialmente el examen de ingreso a la universidad, la que genera que los estudiantes consideren que solamente lo que pueda aparecer en el examen tiene valor, esperen respuestas inequívocas, sientan que cualquier debate es una pérdida de tiempo y piensen que el profesor debe limitarse a seguir los contenidos del currículo. Elton y Laurillard $(1979,23)$ creen que "la manera más rápida de cambiar la manera de aprender de los estudiantes es cambiar el sistema de evaluación".

Lo anterior no es exclusivo de los estudiantes chinos. Biggs (1999) nos recuerda que también en Occidente se visualiza a la enseñanza universitaria como transmisión de información y esto "es aceptado de manera tan generalizada que los sistemas de impartición y evaluación de todo el mundo se basan en ella" $(1999,41)$. También en Occidente es frecuente suponer que el profesor es el experto en conocimientos. Watkins y Biggs (1996) sostienen que el aula china está centrada en el docente pero también en el estudiante ya que los profesores van creando el proceso de enseñanza con sofisticación, orquestan el involucramiento de los estudiantes en clases numerosas y despliegan variedad de estrategias para lograr que sus estudiantes comprendan. 


\subsection{El silencio en el aula}

A diferencia del supuesto generalizado en Occidente de que hablar promueve el pensamiento y el aprendizaje, los estudiantes asiáticos suelen ser silenciosos en clase y no desean hablar en público (Li, 2012). De hecho, creen que hablar interfiere con el pensamiento y no comparten la idea de que la competencia oral se equipare con la inteligencia.

En Occidente, la valorización del habla es un legado de la Grecia antigua y los juicios que requerían una argumentación verbal. Desde muy pequeños se nos enseña a verbalizar los pensamientos y sentimientos. Ocurre, según Li (2012), que ninguna de las tres tradiciones espirituales influyentes en China (taoísmo, budismo y confucianismo) enfatizó el papel del habla. El taoísmo valora la armonía como sabiduría infinita: sin la necesidad de hablar, como se expresa en la frase de Lao Tze: "aquellos que comprenden no hablan, los que hablan no comprenden". El budismo promueve la meditación como medio de tranquilizar la mente y liberarse de los deseos para alcanzar la iluminación personal. Además, para Hwang (1999), en la sociedad agrícola tradicional china, si bien es cierto que una persona tenía oportunidad de interactuar con extraños en vínculos instrumentales, era casi imposible involucrarse en grupos sociales por fuera del clan familiar. Como consecuencia, las relaciones que rodeaban al sujeto estaban caracterizadas por la intimidad pero bajo una estructura jerárquica. De hecho, los chinos son reticentes a hablar con extraños y rara vez inician una conversación con alguien que no conocen (de ahí se deriva la importancia de los intermediarios en cualquier negociación con chinos). Esta es la razón por la que no es demasiado importante en China desarrollar habilidades sociales necesarias para hablar con extraños. La tradición occidental de hablar directamente, debatir y estar en desacuerdo no tiene lugar en un sistema interprersonal jerárquico de esta naturaleza (Li, 2012).

Un estudio de Littlewood (2000) desde una visión contraria a la determinación cultural, argumenta que las características asociadas a los estudiantes chinos como la baja participación en clase son consecuencia de los modelos educativos a los que están acostumbrados más que al propio deseo de los estudiantes. Condujo una encuesta sobre las actitudes hacia la clase de inglés en ocho países de Asia Oriental y en tres países europeos. Halló que la mayoría de los estudiantes de todos los países involucrados en el estudio cuestionan el aprendizaje basado en la transmisión de conocimiento y en la autoridad. La mayoría de los estudiantes (incluídos los chinos) desearía poder participar activamente y tiene una inclinación positiva hacia el trabajo interactivo y significativo.

\subsection{Relación profesor-alumno}

El respeto a los profesores es altamente valorado en la tradición confucianista y está ligado a los valores de sinceridad y humildad (Li, 2012). En los Anales de primavera y otoño, Confucio refiere a las dos actitudes correctas de todo estudiante: sumisión a la autoridad de los padres, mayores y superiores y sumisión a las costumbres de la sociedad. De acuerdo al código de conducta social de Confucio (Wu Lun), existen "Cinco relaciones básicas": gobernador y ministro, padre e hijo, marido y mujer, hermano mayor y hermano menor y la relación entre amigos. El superior tiene la obligación de proteger, mientras que el inferior le debe lealtad y respeto. La armonía solamente se logra si cada uno cumple con los requerimientos de su rol.

Si bien pocos autores se animarían a contradecir estos fundamentos culturales, existen versiones matizadas. Un estudio cualitativo de Xiao (2006) con estudiantes chinos de inglés en una universidad irlandesa muestra que solamente los docentes competentes y considerados con sus alumnos merecen un respeto sincero, mientras que los profesores poco preparados, que demuestran poco interés por sus estudiantes o no planifican suficientemente sus clases no 
serán respetados por aquellos. Por lo tanto, el respeto genuino hacia el docente se basa en la calidad de éste último y en su consideración hacia los estudiantes y no en la simple asimetría de la relación profesor-alumno. Por lo tanto, el respeto a los profesores no significa obediencia, docilidad y falta de pensamiento crítico, como se piensa generalmente.

En la tradición confucianista, un profesor no sólo debe impartir conocimiento sino también transformar al joven en una persona con una consciencia social elevada e inculcarle un código de vida que sea aceptado por los mayores. Esto reflejaría la manera holística que tienen los chinos de ver la enseñanza, que se refiere no sólo a los aspectos cognitivos sino también a los afectivos y morales (Li, 2012). Es preciso mencionar que en China existe una gran interacción entre el profesor y sus estudiantes fuera de clase que dista mucho del vínculo formal que uno puede observar en el aula. Es cierto que la relación docente-alumno está tallada sobre la base de la relación padre-hijo pero es justamente debido a esto que los profesores no sólo deben impartir conocimiento específico sino también ocuparse del bienestar de sus estudiantes.

\subsection{El lugar de la memoria en el aprendizaje}

Los estudiantes chinos son tradicionalmente considerados "memorizadores automáticos", lo que haría suponer que emplean un enfoque superficial de aprendizaje caracterizado por actividades de bajo nivel cognitivo y simple reproducción del conocimiento. Sin embargo, los mismos estudiantes presentan un elevado rendimiento académico, lo que da lugar a la llamada "paradoja del estudiante chino" (Watkins y Biggs, 1996). Según Sánchez Griñán (2009) esta paradoja se resuelve en el hecho de que los estudiantes chinos emplean estrategias cognitivas profundas, al mismo tiempo que repiten y memorizan: "la memorización como modo de comprensión, el recitado al servicio de la reflexión y la interiorización” (pág. 3).

Cuestionarios y entrevistas a estudiantes chinos sobre la manera en que enfrentan las tareas académicas han arrojado que la memorización puede estar ocurriendo junto con intentos por alcanzar una comprensión del material (Kember y Gow, 1989). En la cultura china, memorizar y comprender pueden entonces no ser elementos separados en el proceso de aprendizaje sino procesos conectados e interdependientes. Este método de aprendizaje se ha denominado "memorización con entendimiento". La comprensión puede facilitar la memorización y esta última es precursora del conocimiento profundo (Marton et al, 1996). De hecho, puede ocurrir que la memorización nunca haya sido visualizada como un fin en sí misma sino como un preludio para una comprensión más profunda del material.

Conviene citar a Biggs (1999) en un pasaje interesante a propósito del mito del aprendizaje al pie de la letra de los estudiantes chinos.

"Aprender varios millares de caracteres de uso común requiere más memorización que aprender las veintisiete letras del alfabeto castellano. Sin embargo, en esas circunstancias, la memorización está al servicio de la comprensión. Usted aprende un sistema de comunicación con el fin de comunicarse comprendiendo. (...) La repetición se utiliza también como estrategia para asegurar un recuerdo correcto y aquí opera a favor del significado, no contra él" $(1999,163)$.

Tang (1991) llama a este tipo de utilización estratégica de la repetición "memorización profunda", que no tiene que ver con el aprendizaje memorístico ni con la utilización de un enfoque meramente superficial. Si bien en Occidente existe una distinción entre aprendizaje memorístico y significativo (Ausubel, 1968), no es fácil en nuestra cultura diferenciar el aprendizaje memorístico del repetitivo. El aprendizaje memorístico es el que se realiza sin comprensión del contenido pero no es lo que hacen los estudiantes chinos ya que cuando utilizan la repetición no lo hacen para reducir la complejidad sino para asegurarse el poder 
retener la información. Si la comprensión se combina con la capacidad de recordar, hay altas chances de presentar un buen rendimiento en los exámenes porque éstos siempre implican la necesidad de memorizar información.

La repetición y memorización tienen relación también con la valoración del esfuerzo, que los estudiantes chinos parecen aceptar con más facilidad que los occidentales. Para algunos autores (Chen y Uttal, 1988) esto quizá tenga orígenes culturales ya que el confucianismo considera que el hombre es maleable y la falta de habilidad puede compensarse con el esfuerzo y la perseverancia. En el desempeño académico, la falta de habilidad innata no es excusa para rendirse ya que siempre se puede compensar mediante el esfuerzo. Para Confucio toda persona es educable:

"Es por su naturaleza los hombres son casi iguales pero con la experiencia crecen y se diferencian. Si vemos que otro hombre se esfuerza una vez, nosotros tenemos que esforzarnos cien veces más, si vemos que alguien se esfuerza cien veces, nosotros tendremos que esforzarnos mil veces. Si alguno se esfuerza en este camino, se volverá listo aunque sea tonto y fuerte aunque sea débil". (El justo medio, XX, Anales)

\section{Reflexiones a partir de una experiencia con estudiantes chinos de español como lengua extranjera en Uruguay}

En el marco de un programa conjunto interuniversitario, se interrogó a 42 estudiantes de una provincia del noreste de China, quienes se encuentran estudiando español como lengua extranjera en una universidad privada de Uruguay. Los estudiantes han cursado en su país los primeros dos años de la Licenciatura en Español y cursan el segundo semestre del tercer año de su carrera en Uruguay. En China, los estudiantes cursaron asignaturas de español junto con otras de política, inglés y literatura china. Las asignaturas de español se distribuyen entre las que son dictadas por profesores extranjeros (nativos de español) y las que son dictadas por profesores chinos (gramática y lectura fundamentalmente). El método de enseñanza de español en China es el de gramática-traducción, método que aunque desarrollado por alemanes y extendido en Europa y América desde 1840, su origen se remonta a la forma en que se estudiaba latín y su finalidad era la traducción de textos mediante un sistema de instrucción que se realiza básicamente en la lengua materna de los estudiantes (en este caso, chino mandarín).

Los materiales y libros de estudio, los objetivos del curso y hasta las evaluaciones, son muy distintos de lo que los estudiantes conocen en su larga experiencia dentro del sistema educativo chino. En Uruguay, el método de enseñanza de lenguas es fundamentalmente comunicativo, método en el cual se da importancia a la interacción como medio y como objetivo final del aprendizaje de la lengua y el método de enseñanza de lenguas a través de contenidos. El docente promueve el trabajo en grupo, el aprendizaje por tareas, la modalidad autónoma de aprendizaje y los recursos didácticos más allá del libro de texto (Internet, periódicos, películas, música). Un punto fundamental del método comunicativo de lenguas es la creencia de que la manera en que la lengua funciona es más importante que el conocimiento de su forma y estructura.

Se formularon dos preguntas escritas a ser respondidas anónima e individualmente por cada estudiante mientras finalizaban el segundo semestre en Uruguay.

1) ¿Has cambiado tu manera de estudiar español luego de llegar a Uruguay? Si respondes afirmativamente: ¿De qué manera has cambiado tu manera de estudiar desde que estudias en Uruguay?

2) ¿Qué diferencias hay entre las clases de español en China y las clases de español en Uruguay? 
En términos generales, los estudiantes chinos a quienes se les formularon estas preguntas prefieren un estilo de enseñanza y de aprendizaje más centrado en el estudiante, a pesar de su bagaje cultural del cual se esperaría una predilección por las clases centradas en el docente. Consideran el aprendizaje independiente del libro de texto y del énfasis en el pensamiento crítico, aspectos fuertes de su experiencia en Uruguay. Parece existir, asimismo, una preferencia por un estilo más global y abierto de aprendizaje. Los estudiantes señalan en China clases centradas casi exclusivamente en el libro de texto y en la gramática, lo que se refleja en las siguientes afirmaciones:

"En China, en clase seguimos siempre el libro pero en Uruguay, podemos hablar de otras cosas que nos ocurren, relacionadas con lo que aprendimos o de nuestra vida o alguna experiencia actual".

"Acá estudio menos a través del libro y estudio más por la radio, periódico y hablando con la gente. En China estudio gramática, acá puedo estudiar historia, cultura y música".

"En Uruguay es importante que preste atención en clase. La comprensión es lo más importante. En China, si pierdo algunas clases siempre puedo encontrar el conocimiento en el libro".

Como ilustran estas respuestas, en Uruguay aparecen alternativas al libro de texto (radio, televisión, periódico e incluso amigos), las cuales son integradas al esquema de recursos de aprendizaje. El estilo de enseñanza en China es más centrado en el docente como fuente de conocimiento y transmisión del mismo.

Chen (1990) sostiene que los estudiantes chinos están motivados a aprender pero no a tomar riesgos, empleando un estilo convergente ya que tienden a creer que sólo una respuesta es la correcta. Los estudiantes que fueron interrogados parecen percibir que los profesores les exigen dar respuestas que pueden tener varias soluciones.

"Los profesores uruguayos quieren que los estudiantes piensen más. Al contrario, en China, los profesores esperan que los estudiantes anoten lo que ellos dicen".

"Acá en clase, es importante que propongamos nuestras ideas y los profesores pueden cambiar la forma de dar clase según nuestras necesidades".

"En China, la profesora da la respuesta perfecta a una pregunta pero acá no hay respuestas fijas. Tengo que pensar y expresar mi opinión sobre el tema, por ejemplo sobre una noticia o una publicidad".

Los estudiantes resaltan incluso la necesidad que tienen en Uruguay de seleccionar ellos mismos qué es lo más importante ya que el profesor no realiza esa priorización inicial.

"En China los profesores siempre escriben mucho en la pizarra y los estudiantes copian y lo que está en la pizarra es casi todo lo que explica el profesor en clase. En Uruguay, los profesores escriben en cualquier lugar de la pizarra y muchos no escriben nada. Los estudiantes tienen que decidir qué es importante".

La posibilidad de equivocarse y plantear dudas es otra de las diferencias con China. Es frecuente en China que las preguntas se realicen una vez finalizada la clase ya que difícilmente un estudiante planteará sus dudas frente a todo el grupo.

"En China si tienes algunas dudas, no puedes preguntar directamente, necesitas esperar cuando termina la clase. En Uruguay si tienes preguntas puedes preguntar en cualquier momento y los profesores te explican con paciencia". 
Si los estudiantes asiáticos adoptan actitudes pasivas en clase, esto puede deberse más a aspectos situacionales y contextuales que a disposiciones inherentes de los estudiantes.

La frase de una estudiante es ilustrativa:

"En China, la profesora es muy seria aunque cuida de nosotros. A veces tengo miedo de cometer errores y no quiero hablar mucho en clase. Acá, hablo mucho más en clase aunque me equivoque".

Como vimos, puede ocurrir que la memorización nunca haya sido visualizada como fin en sí misma sino como un preludio para una comprensión más profunda (Marton et al., 1996). Ocurre que los estudiantes pueden tener una preferencia por comprender pero reconocen que normalmente los exámenes como el gao kao requieren reproducir el material de memoria. Podría pensarse que la predilección por la memorización no se trate necesariamente de una tradición cultural arraigada que nace con el confucionismo sino de una consecuencia contextual de las evaluaciones. No hay que olvidar que el propio Confucio consideraba el aprendizaje como algo profundo: "aprender sin pensar es inútil, pensar sin aprender es peligroso" (Anales de primavera y otoño).

Lo anterior se refleja en algunas de las frases de los estudiantes:

"En China tenemos que aprender de memoria todos los textos de Español Moderno, algunos de ellos son muy largos pero acá la memoria no importa tanto".

"En China, lo más importante es la gramática y el libro y recordar todo lo que aprendemos. En Uruguay, lo importante no es recordar todo sino que entendamos más".

" (...) cuando estaba en China todos los días hacía ejercicios del libro y leía los textos una y otra vez. Estos días estoy leyendo un libro que se llama La sombra del viento. Cuando encuentro palabras o frases que no entiendo, puedo buscarlas en Internet o preguntarle a alguien".

Mientras que algunos estudiantes parecen valorar la importancia dada a la producción oral y las prácticas comunicativas, otros resaltan la importancia de la memorización e incluso se quejan de la falta de atención a estos aspectos en clase por parte de los profesores en Uruguay:

"En China, todo el día estudiamos español recitando los textos, la gramática, las palabras. Acá prestamos más atención al estudio de oral y audición lo que me parece bien pero falta un poco de práctica y repetición".

"En China gasto mucho tiempo en estudiar gramática y memorizar las palabras pero desde que llegué a Uruguay, me importa más oral y audición. Quiero hablar con mis amigos uruguayos y conocer la cultura. Me parece que es una muy buena manera de practicar oral y más fácil y rápido. Sin embargo, para el examen, la manera de estudiar no cambió. Recuerdo de memoria".

"Para los uruguayos no es difícil recitar en lengua extranjera porque son parecidas las lenguas entre ellas. En cambio el chino no es igual y es muy diferente del español. Por eso necesitamos recitar con más esfuerzo. Eso me gusta de China".

Podemos ver que los estudiantes pueden tener una manera preferida de acercarse al estudio pero estas maneras son también influenciadas por la percepción que tienen sobre la naturaleza de la tarea académica, el modo de evaluación o los métodos de enseñanza y aprendizaje presentes. La enorme presión que tienen los estudiantes chinos frente a exámenes torna difícil resistir la tentación de utilizar maneras de aprender que ayuden al éxito en las evaluaciones. Como sostienen Kember y Watkins (2010): 
"Si los estudiantes chinos son genuinamente observados como estudiantes memorísticos y que emplean una aproximación superficial al aprendizaje, esto no es una manifestación de una predisposición cultural inherente a las características del aprendiente chino. Es, en realidad, una respuesta a las percepciones de los factores culturales en el ambiente de enseñanza y aprendizaje y de la influencia de las expectativas sociales y culturales" $(2010,174)$.

Las siguientes frases son ilustrativas de este punto de vista:

"En China, sólo estudio para el examen, acá estudio la lengua y cultura para comunicarme".

"En China, las clases se enfocan más en el léxico, la gramática. Todas las clases sirven para que los alumnos puedan tener buenas notas en los exámenes de español. En Uruguay, el español sirve para hablar y entender".

"En China, las respuestas de los exámenes son siempre una sola pero en Uruguay hay preguntas abiertas".

"En China, antes de los exámenes puedo saber qué tengo que preparar. Ahora en Uruguay, no sé lo que tengo que estudiar y cosas que preparo no van en el examen".

Los exámenes en Uruguay son más flexibles que en China. No sabemos qué se va a preguntar en el examen, por eso tenemos que estudiar todo y no sólo de memoria".

El tiempo de clase es mucho más breve en Uruguay que en China porque en su tierra reciben además de los cursos en español, clases de pensamiento marxista, inglés y literatura china. El horario suele extenderse desde las 8 a las 18 horas con pausas para el almuerzo y muy breves descansos entre clase y clase. En Uruguay, los estudiantes asisten a clases por la tarde, recibiendo un total de 19,5 horas semanales de clase (misma carga horaria que en China para las materias específicas de español como lengua extranjera). Esta variación en horario de clase tiene consecuencias, siendo la principal de acuerdo al relato de los estudiantes, el contar con mayor tiempo libre ya sea para realizar actividades recreativas como para utilizar recursos de aprendizaje de español diferentes a los relacionados estrictamente con el curso.

"No leo muchos libros desde que llegué a Uruguay, pero veo películas y telenovelas en español. Tengo mucho tiempo libre, puedo utilizar el tiempo libre para aprender la cultura y conocer otro aspecto sobre la lengua, por ejemplo, la música y programas de Argentina y las costumbres de los latinoamericanos".

"Cuando estaba en China tenía muchos deberes sobre gramática pero acá tenemos deberes que tienen que ver con hacer entrevistas, presentar noticias, etc".

"En China hacía muchos ejercicios y recordaba palabras de memoria. Acá no hago tantos ejercicios pero leo y veo películas. En Uruguay, siempre conozco nuevas palabras o expresiones porque las escucho y leo".

El énfasis en la gramática y la forma es otra diferencia que mencionan los estudiantes. En China, la mayor cantidad de horas semanales de español (16) son reservadas a la gramática, con clases dictadas mayormente en chino y sólo 2 o 4 horas por semana se dedican a oral y audición con la presencia de un profesor extranjero. Es cierto que también en Uruguay hay clases de gramática, que incluso utilizan la misma serie de libros que los estudiantes emplearon en China durante los primeros dos años de la carrera (Español Moderno). Sin embargo, 
importantes variaciones se realizan durante el dictado de dichas clases: actualizar vocabulario, intentar acercar el contenido de los textos a la experiencia de los estudiantes de modo de personalizarlos, búsqueda de similares maneras de expresar la misma idea e integración de otros recursos materiales además del texto del curso.

"En Uruguay la forma no es tan importante, lo importante es el contenido. Por ejemplo, si escribimos a mano o en computadora es lo mismo pero en China la forma es muy importante. Hay que escribir así, de una sola manera".

"En China la gramática es muy importante porque tenemos un examen de gramática pero en Uruguay la gramática no es tan importante como hablar y entender".

El cambio de contexto educativo y de metodologías de enseñanza de lenguas podría generar cambios en los estilos de aprendizaje de los estudiantes. Los estudiantes aprenden las reglas del ambiente de aprendizaje en que se encuentran, comportándose de acuerdo a ellas. Una vez que migran de un lugar a otro, moviéndose en un contexto educativo diferente, encuentran distintas reglas y se adaptan a ellas.

"En China siempre pensamos en chino para aprender español. Acá pensamos en español".

"En China, lo más importante es practicar y trabajar. Tanto en clase como fuera de clase, tienes que practicar el presente del subjuntivo o el imperativo una y otra vez. Pero acá creo que hablamos más".

"Cuando leo en Uruguay me enfoco más en el conjunto del texto pero en China el fragmento es lo más importante".

\section{Reflexiones finales}

"Si bien, conceptos corrientes como la obediencia y la falta de cuestionamiento a la autoridad de los estudiantes chinos son repetidos tan frecuentemente" que resultaría difícil negar que estén basadas en alguna forma de realidad, la pregunta que se plantea Littlewood (2000) es si esta "realidad" está basada en las preferencias y disposiciones de los estudiantes, quienes solamente desean escuchar y obedecer, o si los estudiantes simplemente se adaptan a las características de un determinado contexto educativo.

El primer año de enseñanza en China suele ser una experiencia deslumbrante. Para los estudiantes que no pertenecen a las ciudades más cosmopolitas de China (Beijing, Shanghái, Hong Kong), se trata probablemente de la primera vez que tienen un profesor extranjero y quizá, la primera vez que conocen a uno personalmente. La tentación de sentirse casi una celebridad pronto se compensa con las dificultades al enseñar estudiantes que no comparten la lengua materna del docente y que tienen particularidades a la hora de dirigir su proceso de adquisición de la lengua. Conocer a los estudiantes resulta esencial, tanto para proporcionar efectivos y apropiados métodos para enseñar español como también para aumentar su motivación y disminuir la ansiedad en el aprendizaje.

Mi propia experiencia con estudiantes chinos me ha llevado a cuestionar la afirmación de que los estudiantes chinos disfrutan de escuchar sin participar, memorizar y obedecer al profesor, y muchas de las expresiones de los estudiantes a partir de las preguntas formuladas parecen confirmar en parte esta impresión. El estudio de Littlewood (2000) indica claramente que el estereotipo del estudiante asiático como "oyente obediente" no refleja los roles que ellos querrían adoptar en clase: "no ven al docente como una figura de autoridad que no debería ser cuestionada, no quieren sentarse pasivamente en clase recibiendo conocimiento y están apenas de acuerdo con la idea de que el docente debería tener un mayor rol que ellos mismos en evaluar su aprendizaje" ( $p, 2)$. 
Es cierto que parte del buen desempeño académico chino quizá tenga que ver con valores arraigados como la importancia asignada a la educación, el énfasis en el esfuerzo y la práctica, la creencia en la persistencia para llegar al éxito, entre otros. Sin embargo, la importancia de la cultura tal vez haya sido sobredimensionada y haya contribuido al estereotipo del estudiante chino como pasivo y memorizador automático, incapaz de salir de ese rol aunque su situación cambie. Sánchez Griñán $(2008,408)$ resume la impresión de 15 profesores extranjeros sobre los estudiantes chinos, señalando aspectos positivos: "la diligencia, la persistencia, el perfeccionismo y el carácter afectuoso, así como la capacidad para memorizar y el deseo de aprender bien". Sin embargo, es a la hora de poner en práctica una metodología comunicativa de aprendizaje de lenguas cuando surgen los aspectos negativos, como la excesiva atención a la gramática y al texto escrito, la dificultad para comunicarse oralmente y por escrito, poca disposición a trabajar en grupo, excesiva atención a los exámenes y timidez. Muchos de estos aspectos han sido interpretados como productos de la cultura china, en la cual convergen elementos del confucianismo y de la tradición pragmatista que asigna especial importancia al sistema de exámenes y a la traducción (Rossi-Le 1995; Chan, 1999). Contrariamente a estos autores, como vimos, otras miradas (Kee-Kuok, 2004) han sostenido que el aprendizaje es el resultado del modelo de enseñanza en sentido amplio (metodologías de enseñanza de lenguas, métodos de evaluación, tipo de interacción docente-alumno requerimientos de aprobación del curso) y no solamente de aspectos más abstractos como los sistemas culturales o simbólicos.

No necesariamente tenemos que negar la influencia de la cultura en la conducta y los estilos de aprendizaje de los estudiantes. Sin embargo, todavía falta mucho para poder explicar la naturaleza y la extensión de dicha influencia así como para conocer también el efecto que tienen los diferentes entornos educativos en las concepciones acerca del aprendizaje.

\section{Bibliografía}

Ausubel, D. (1968). Educational psychology: a cognitive view. Nueva York: Holt, Rinehart and Winston.

Biggs, J. (1999). Calidad del aprendizaje universitario. Madrid: Narcea.

Chan, S. (1999). The Chinese learner-a question of style. Education and Training, págs. 294-304.

Chen C.S. ; Uttal, D. H. (1988). Cultural values, parents' beliefs, and children's achievement in the United States and China. Human Development, págs. 351-358.

Cheng, X. (2000). Asian students' reticence revisited. System, 28, pág. págs.435-446.

Confucio (1990). Anales. Shangdong: Shangdong Youyi Shushe.

Confucio (2002). Los Cuatro Libros. Buenos Aires: Paidós.

Dörney, Z. (2005). The Psychology of the Language Learner. Individual differences in Second Language Acquisition. New Jersey, Lawrence Erlbaum Associates Publishers.

Elton, L.R.B; Laurillard, D.M. (1979). Trends in Research on Student Learning. University of Surrey, Institute for Educational Technology.

Entwistle, N. J.; Ramsden, P. (1983) Understanding Student Learning. Londres: Croom Helm. Gow, L. K. (1990). Does higher education promote independent learning? Higher Education, 19, págs.307-322.

Hwang, K.-K. (1999). Filial piety and loyalty: Two Types of Social identification in Confucianism. Asian Journal of Social Psychology, 2, págs..163-183.

Kee-Kuok Wong, J. (2004). Are the Learning Styles of Asian International Students Culturally or Contextually Based? Recuperado el 25 de enero de 2013 de International Education Journal: http://iej.cjb.net 
Kember, D. (1989). Cultural specificity of approaches to study. Trabajo presentado en la 6ta Conferencia Anual de la Hong Kong Educational Hong Kong. (págs. 356-363).

Kember, D. (2001). Beliefs about knowledge and the process of teaching and learning as a factor in adjusting to study in higher education. Studies in Higher Education, págs.205-221.

Kember, D. (2010). Approaches to learning and teaching by the Chinese. En M. H. Bond, The Oxford Handbook of Chinese Psychology (págs. 169-185). Oxford: Oxford University Press.

Kinsella, K. (1995). Understanding and empowering diverse learners in ESL classroom. En J. Reid, Understanding Learning Styles in the Second Language Classrooms (págs. 19-33). Nueva York: Heinle \& Heinle.

Kirkbride, Paul; Tang, Sara (1991). Chinese Conflict Preferences and Negotiating Behaviour: Cultural and Psychological Influences. Organization Studies , 12(3), (págs. 365-386).

Littlewood, W. (2000). Do Asian students really want to listen and obey? ELT Journal, (págs. 31-36).

Li, J. (2012). Cultural Foundations of Learning. East and West. Cambridge: Cambridge University Press.

Marton, F.; Dall'Alba, G.; Kun, T.L. (1996) Memorising and understanding: the keys to the paradox? En D. B. Watkins, The Chinese learner: Cultural, psychological and contextual influences (págs. 69-84). Melbourne: Australian Council for Educational Research and the Comparative Education Research Centre.

Melton, C. (1990). Bridging the cultural gap: A study of Chinese students' learning style preferences. RELC Journal, (págs. 29-47).

Nelson, G. (1995). Cultural differences in learning styles. En R. J, Learning styles in the ESL EFL classroom (págs. 3-18). Boston: Heinle \& Heinle.

Neuman, Y. B. (2000). Cultural resources and the gap between educational theory and practice. Teachers College Record (págs. 496-500).

Ramsden, P. (1979). Student learning and perceptions of the academic environment. Higher Education, 8, (págs. 412-425).

Ramsden, P. (1981). A study of the relationship between student learning and its academic context. Tesis de Doctorado no publicada. University of Lancaster.

Rossi-Le, L. (1995). Learning styles and strategies in adult inmigrant ESL students. En J. Reid, Language learning styles in the ESL/EFL classroom (págs. 118-125). Boston Heinle \& Heinle.

Sánchez Griñán, A. (2008). Enseñanza y aprendizaje de español como lengua extranjera en China . Recuperado el 15 de octubre de 2012 de www.tesisenred.net/TDR-0731108-134322/ index_es.html

Sánchez Griñán, A. (2009). Estrategias de aprendizaje de alumnos chinos de español. Recuperado 15 de octubre de 2012 de Marco ELE: www.marcoele.com/descargas/china/ sanchez-grinan_estrategias.pdf

Scarcella, R. (1990). Teaching language minority students in the multicultural classroom. Engelwood Cliffs: NJ: Prentice Hall.

Spizzica, M. (1997). Cultural Differences Within Western and Eastern Education. En H. B. Z. Golebiowski, Academic Communication accross Disciplines and Cultures (págs. 248-257). Melbourne: Victoria University of Technology. 
Tai Hau, K. H. (2010). Chinese students'motivation and achievement. En M. H. Bond, The Oxford handbook of Chinese psychology (págs. 188-204). Oxford: Oxford University Press.

Tang, C. (1991). Effects of two different assessment procedures on tertiary students' approaches to learning. Hong Kong: University of Hong Kong.

Tse, L. (2006). Tao Te Ching: Los libros del Tao. Traducción de Iñaki Preciado. Madrid: Trotta.

Volet, S; Renshaw, P. (1996). Chinese students at an Australian university: Adaptability and continuity. En J. B. D.A. Watkins, The Chinese Learner: Cultural, Psychological and Contextual Influences (págs. 205-220). Hong Kong: The Central Printing Press.

Watkins, D.A.; Biggs, J.B. (1996). The Chinese Learner: Cultural, psychological and contextual influences. Hong Kong: Comparative Education Research Centre; Victoria, Australia: The Australian Council for the Educational Research.

Xiao, L. (2006, 12). Bridging the Gap Between Teaching Styles and Learning Styles: a CrossCultural Perspective. Recuperado el 25 de abril de 2012 de TESL-EJ: http://www.tesl-ej.org/ ej39/a2.pdf

Zhang, L.F.; Sternberg, R.J.; Rayner, S. (2012) Handbook of Intellectual Styles. Preferences in cognition, Learning, and Thinking. New York: Springer Publishing Company.

Zhang, L. F. (2000). University students'learning approaches in three cultures: An investigation of Biggs's 3P model. The Journal of Psychology, 134(1), (págs. 37-55).

Fecha de recibido: 04/02/2013

Fecha de aceptado: 30/05/2013

${ }^{*}$ Doctora (Cand.) en Educación, Universidad ORT Uruguay. Master en Educación, Universidad ORT Uruguay. Licenciada en Psicología. Postgrado en Recursos Humanos, Universidad Católica del Uruguay. Profesora de Inglés como Lengua Extranjera, Certificate TESOL, Trinity College of London. Coordinadora de Proyecto Conjunto, Universidad ORT Uruguay - Universidad Pedagógica de Harbin (China). 